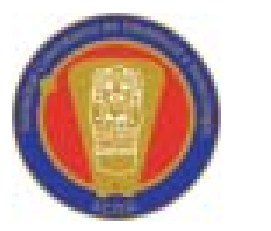

\title{
EDITORIAL
}

\section{EL AVANCE ESPERADO DE LAS PUBLICACIONES CIENTÍFICAS COLOMBIANAS}

\author{
Javier H. Eslava, M.D., MSc, MDU.*
}

«Cuando un arquero falla su tiro busca el error en él mismo y no en el blanco; trabaja para mejorar y acertar en el próximo intento. Si a esto agregamos que cada tarea que realizamos es un autorretrato, llegaremos a la conclusión de que la nación que hoy en día tenemos lleva el autógrafo de sus autores: los líderes que han escrito nuestra historia»

Miguel Ángel Cornejo ${ }^{1}$

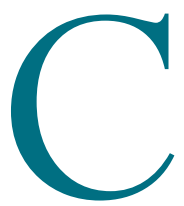
olombia se ha caracterizado por tener desde hace mucho tiempo publicaciones científicas. La Revista de la Facultad de Medicina de la Universidad Nacional, por ejemplo, publicó su primer número en junio de $1932^{2}$ y alcanzó a figurar en la Librería Nacional de Medicina desde la misma fecha, con inclusión al Index Medicus desde 1965 a 1981. ${ }^{3}$ También se encontró la Revista de la Facultad de Odontología entre los periodos de 1963 a $1973^{4}$ y Colombia Médica desde 1939 a 1946, con un periodo de suspensión entre 1940 y 1943, y como Colombia médica (Cali-Colombia) desde 1990. ${ }^{5}$

Con la llegada de las especialidades medico-quirúrgicas se incrementó el número de publicaciones periódicas con la participación de las recién creadas sociedades científicas. De esta manera, por ejemplo, se encontraron la Revista Colombiana de Obstetricia y Ginecología, y la de la Sociedad Colombiana de Pediatría y Puericultura. La primera con figuración en el Index Medicus y Medline entre 1965 y 1971, con reubicación especial en Medline entre 1973 y 1978, ${ }^{6}$ y la segunda desde octubre de 1941, con ingreso al Index Medicus sólo para abril de 1965.7

* Profesor Asociado. Facultad de Medicina. Universidad Nacional de Colombia.
En total a la fecha son 12 las Revistas que se encuentran en Medline con la palabra clave «Colombia», entre las que además se encuentran las revistas Análisis Político (del Instituto de Estudios Políticos y Relaciones Internacionales de la Universidad Nacional de Colombia, desde 1987), el Boletín Mensual de Estadísticas del Departamento Administrativo Nacional de Estadística (entre 1953 y 1984), el Boletín Socioeconómico de la Universidad del Valle (entre 1985 y 1999), Coyuntura Económica de la Fundación para la Educación Superior y el Desarrollo (desde 1971) y la Revista de Salud Pública (Bogotá-Colombia) del Instituto de Salud en el Trópico de la Universidad Nacional de Colombia, desde 1999, e incluida en el Index Medicus desde el 2003. ${ }^{8}$

Como es evidente, los protagonistas en la base de datos de la Librería Nacional de Medicina han sido 2 universidades públicas, 2 sociedades científicas y una Fundación. Actualmente figuran en bases de datos internacionales 10 revistas, 3 de ellas en Medline: Biomédica (Revista del Instituto Nacional de Salud), la Revista de Salud Pública (de la Universidad Nacional de Colombia) y Colombia Médica (de la Universidad del Valle). Las otras siete se encuentran en PsyINFO, EconLIT, Social Science Citation Index Arts and Humanities y Agropecuaria. ${ }^{9}$

En total, en proceso de clasificación y con calificación según el reporte de Colciencias para el año 2004 y hasta marzo del 2005 se encuentran 88 revistas colombianas que han cumplido con una serie de requisitos ante Colciencias, pero que de las cuales, solamente han superado el proceso de 
indexación en las bases de datos mencionadas, diez. Es decir, 78 revistas colombianas de diferente calidad, y de diferente perfil deberían estar adelantando gestiones para ser incluidas en las bases de datos de acceso y consulta mundial. En el Portal de Revistas Colombianas en Ciencias de la Salud, al cual se accede a través de Bireme, se encuentran 56 revistas, con diferente nivel de calidad y audiencia. ${ }^{10}$ Algunas de estas revistas ofrecen la posibilidad de consulta en texto completo de sus volúmenes. Adicionalmente, otras han aplicado y calificado al Proyecto Scielo, cuya intención es proveer acceso a una biblioteca virtual electrónica en texto completo, como parte integral del proyecto FAPESPBIREME, con 18 tópicos diferentes, y en el que hasta la fecha están incluidas seis revistas: la Revista de la Sociedad Colombiana de Obstetricia y Ginecología, la Revista de Salud Pública, la revista Colombiana de Cardiología, Revista Colombiana de Cancerología, Revista Colombiana de Gastroenterología y Revista Colombiana de Neumología. ${ }^{11}$

El esfuerzo nacional por tratar de hacer que las revistas colombianas alcancen reconocimiento internacional es evidente en el aval dado por Colciencias a las 88 revistas que se mencionaron atrás. De éstas, 71 se encuentran en Categoría C, 7 en Categoría B y 10 en categoría A (Gráfica 1). Nuevamente, se aprecia el papel de las universidades públicas y privadas, de las Fundaciones y el de las asociaciones científicas y los Institutos en la circulación y mantenimiento de estas revistas. Sin embargo, ocho revistas de las asociaciones científicas y cuatro de los Institutos no han ingresado a los índices internacionales, que les permitan calificar en categoría A1 o A2.

Es evidente que la fortaleza institucional de las Universidades les permite tener tanto comités editoriales con personas que publican frecuentemente y figuran por sí mismos en los índices internacionales, como pares académicos que les permiten calificar de mejor manera la calidad de los productos que se publican. Adicionalmente cuentan líneas de investigación, que generan productos susceptibles de ser publicados en dichos espacios.

A pesar de esto, se ha dado un círculo vicioso entre investigadores y revistas, en el que ellos no quieren publicar en las revistas nacionales que no figuran en los índices, y las revistas por su parte, no pueden aplicar a ser consideradas para figurar en los índices, pues carecen de la suficiente cantidad de artículos originales pertinentes y de buena calidad. Ejemplo de ello se referencia en la cantidad de ensayos clínicos publicados que figuran en la base de datos de Medline (5\%), y los experimentos que figuran en la base de datos Lilacs (1\%), ${ }^{12}$ lo que sugiere que los investigadores prefieren $\mathrm{pu}$ -

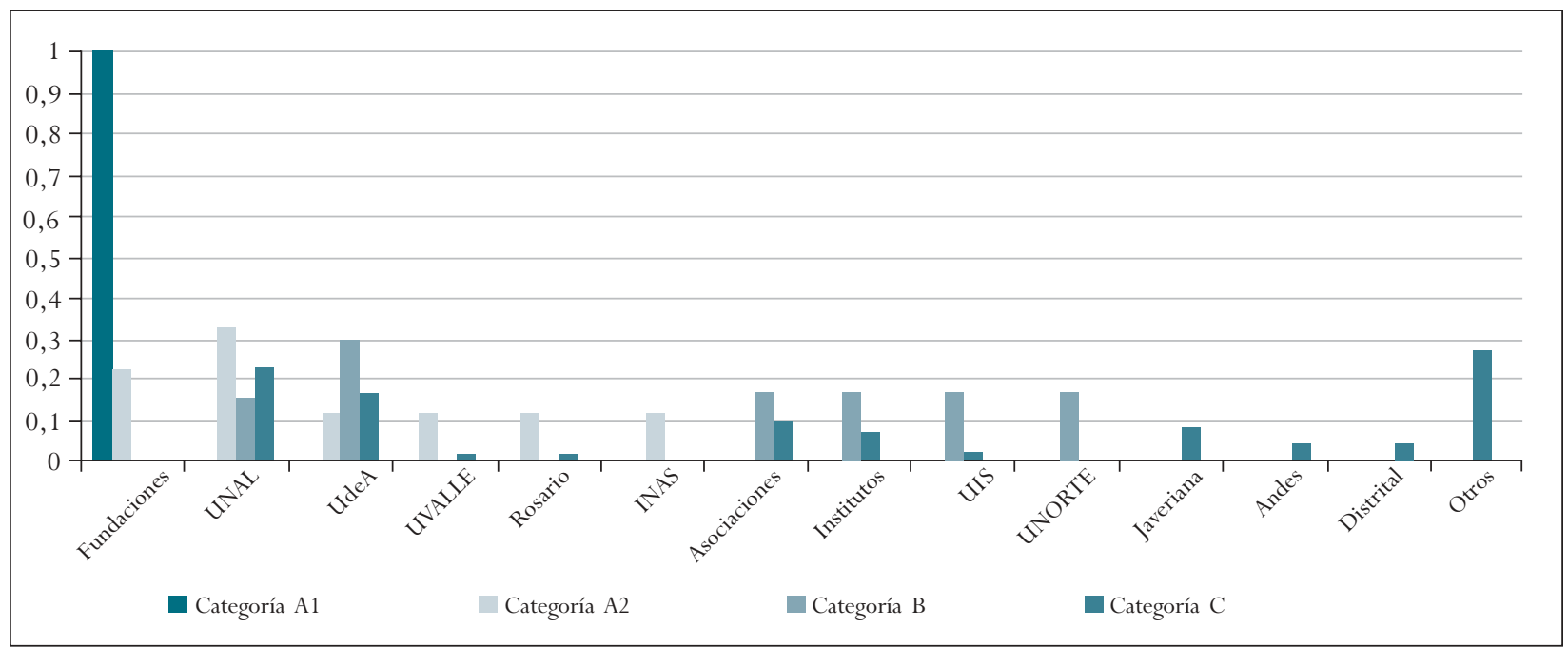

Gráfica 1. Proporción de revistas calificadas por Colciencias, según institución y categoría, Publindex. Fuente: a partir de Publindex, junio 2002 a marzo 2005, Colciencias. 
blicar sus ensayos clínicos en revistas que figuren en índices como Medline, Embase, etc., y no como Lilacs que no cuenta con reconocimiento mundial, sino solo latinoamericano.

Las asociaciones académicas han tratado de fortalecer la investigación dentro de sí mismas, generado espacios en los que se promueve la investigación de sus asociados, bien sea desde la academia o no, como son los premios nacionales de las respectivas asociaciones, para proyectos terminados o protocolos en curso y la realización de cursos de investigación. Sin embargo, este esfuerzo no ha sido suficiente para obtener el volumen y la calidad de publicaciones requerido para figurar en los índices.

Lo que queda por venir es más difícil. Cada día, los autores se relacionan más con sus pares internacionales y con las revistas que éstos leen, de tal suerte que quieren publicar menos en las revistas colombianas. Por su parte, a las universidades les compete mejorar sus publicaciones haciendo uso de sus fortalezas, como la investigación y la disponibilidad de pares académicos reconocidos; las asociaciones deben estimular en mayor medida la investigación y publicación por sus asociados; generar líneas de investigación con capacidad de financiación propia en investigación clínica y básica; y establecer alianzas estratégicas con la academia, para apalancarse mutuamente en la generación de nuevas y mejores investigaciones publicables. Como soporte de los anterior está el hecho que de los 1.283 grupos de investigación reconocidos por Colciencias, 1.150 estaban relacionados con las Universidades, 53 con Institutos, y sólo uno con una Asociación. ${ }^{13}$

Finalmente, a los investigadores nos compete también hacer el esfuerzo de apoyar nuestras revistas, siempre y cuando estén adelantando las gestiones de indexación, publicando nuestros productos de buena calidad en ellas, participando como revisores pares, y dando sugerencias y apoyo constructivo, si en realidad queremos que las revistas colombianas tengan el avance que esperamos.

\section{REFERENCIAS}

1. Cornejo, Miguel. El futuro es hoy. En Cornejo, Miguel. Todos los secretos de la excelencia. Editorial Grijalbo S.A. México D.F. 1995. p83

2. Diaz-Llano R. La Revista de la facultad (Editorial). Revista Facultad de Medicina 2004;52(4):1-2.

3. National Library of Medicine, Locutor Plus Database (NML-Locator), diciembre de 2004 [Internet]. http:/ /locatorplus.gov/cgi-bin/Pwebrecon.cgi? DB = local\&v2 = 1\&ti=1,1\&Search_Arg=7507464\&Search _Code $=0359 \& \mathrm{CNT}=20 \& \mathrm{SID}=1$

4. NLM-Locator, diciembre de 2004, [Internet] http:// locatorplus.gov/cgi-bin/Pwebrecon.cgi?DB = local\&v2 $=1 \& \mathrm{ti}=1,1 \&$ Search_Arg $=7505968 \&$ Search_Code $=$ $0359 \& \mathrm{CNT}=20 \& \mathrm{SID}=1$

5. NLM-Locator, diciembre de 2004, [Internet] http:// locatorplus.gov/cgi-bin/Pwebrecon.cgi? $\mathrm{DB}=$ local \&v2 $=1 \& \mathrm{ti}=1,1 \&$ Search_Arg $=9212578 \&$ Search_Code $=$ $0359 \& \mathrm{CNT}=20 \& \mathrm{SID}=1$

6. NLM-Locator, diciembre de 2004 [Internet] http:// locatorplus.gov/cgi-bin/Pwebrecon.cgi?DB = local\&v2 $=1 \& \mathrm{ti}=1,1 \&$ Search_Arg $=0404263 \&$ Search_Code $=$ $0359 \& \mathrm{CNT}=20 \& \mathrm{SID}=1$

7. NLM-Locator, diciembre de 2004 [Internet] http:// locatorplus.gov/cgi-bin/Pwebrecon.cgi?DB = local\&v2 $=1 \& \mathrm{ti}=1,1 \&$ Search_Arg $=0417463 \&$ Search_Code $=$ $0359 \& \mathrm{CNT}=20 \& \mathrm{SID}=1$

8. Búsqueda en National Library of Medicine-Pubmed, diciembre de 2004 [Internet] http://www.ncbi.nlm.nih.gov/ entrez/query.fcgi? $\mathrm{CMD}=$ search $\& \mathrm{DB}=$ journals

9. Instituto Colombiano para el Desarrollo de la Ciencia y la Tecnología 'Francisco José De Caldas' Colciencias. Indice Bibliográfico Nacional - Publindex. Bogotá: COLCIENCIAS, 2004:1-5.

10. Biblioteca Virtual en Salud, Organización Panamericana de la Salud, Organización Mundial de la Salud. [Internet] [http://www.col.ops-oms.org/iah/ portal.htm. Diciembre de 2004

11. Scielo-Colombia, Biblioteca Virtual en Salud, Bireme. [Internet] http://scielo-co.bvs.br/scielo.php?script $=$ sci_alphabetic\&lng $=$ es\&nrm $=$ iso

12. Eslava J. Estado del Arte de la Investigación en Anestesia. Revista Colombiana de Anestesiología 2002;30: 53-59.

13. Instituto Nacional de Ciencia y Tecnología, Francisco José de Caldas, Colciencias. Listado de Grupos Reconocidos a Septiembre 3 de 2004. 